O8 (continued)

inexpensive strategy for promoting some health behaviors.

Funding: Academy of Nutrition and Dietetics Foundation.

\section{Application of Critical Thinking Skills to Provocative Topics in Nutrition: A Course for Undergraduate Students}

Elizabeth Ruder,PhD, RD, MPH, eruder@pitt.edu, University of Pittsburgh, 4051 Forbes Tower, Pittsburgh, PA 15260; L. Martich, RD, LDN

Objective: To describe development and student response to an undergraduate course focused on application of critical thinking (CT) to provocative topics in nutrition.

Target Audience: Senior-level students enrolled in an accredited Didactic Program in Dietetics.

Theory, Prior Research, Rationale: Accreditation guidelines for nutrition/dietetics programs espouse CT, but CT skill-development is often overlooked. We utilize Stage Theory of Critical Thinking Development to design a 3-credit CT course for nutrition/dietetics undergraduates. Description: Application of Critical Thinking to Nutrition Issues at the University of Pittsburgh is organized in two themes: influence of personal and professional bias on CT; and evaluating media in shaping perception of nutrition issues. Theme one topics include personal examination of weight bias, the Health at Every Size movement, and corporate sponsorship within the Academy of Nutrition and Dietetics. In theme two, students review popular media and evaluate portrayal of nutrition issues with special attention to use of statistics and scientific accuracy. Evaluation is achieved through facilitated discussion and written assignments.

Evaluation: Using a 5-point scale, 29 students indicated greatest interest in weight bias (mean $=4.24, \mathrm{SD}=0.87$ ) and facilitated discussion of the role of processed food in development and prevention of obesity (mean $=4.41$, $\mathrm{SD}=0.68$ ). Favorable increases in knowledge (mean= $4.00 / 5.00, \mathrm{SD}=1.00$ ) and ability to navigate provocative topics and identify bias in free-response comments on university-administered course evaluation.

Conclusions and Implications: CT skills are crucial for future nutrition professionals, CT theory suggests development is not automatic, but requires guidance. An initial step in CT development is personal examination, upon which skills are built to develop the accomplished thinker. Students viewed this unique course favorably.

Funding: None.

\section{Impact of a Service-Learning-Based} Community Nutrition Course on Students' Nutrition Teaching Self-Efficacy

Natalie K. Cooke,PhD, nkcooke@ncsu.edu, North Carolina State University, Campus 7624, Raleigh, NC 27695;

S. L. Ash, PhD; J. Nietfeld, PhD; A. Fogleman, PhD, RD, IBCLC; L. S. Goodell, PhD, RD
Objective: The purpose of this study was to determine the effect of a service-learning (SL) course on student self-efficacy (SE) in teaching nutrition in the community.

Target Audience: Students in the experimental group $(n=20)$ were enrolled in a Community Nutrition SL course, and students in the control group were in a Public Health Nutrition non-SL course $(n=63)$.

Theory, Prior Research, Rationale: To build SE, students need to practice their skills in an appropriate setting. Service-learning, a pedagogy combining academic learning with service in the community, is an ideal framework for this preparation.

Description: The SL course consisted of a 6-week pre-SL training designed to increase SE through skills-based training, observation, and practice and a 6-week SL experience in which students taught a pre-packaged curriculum in the community.

Evaluation: Researchers developed the Self-Efficacy in Teaching Nutrition in the Community (SET-NC) survey to evaluate changes in student SE for teaching nutrition in the community. Experimental and control groups took the SET-NC survey three times during the semester. Using time 1 as a covariant, results from a repeated measures ANCOVA indicated the experimental group had significantly higher SE scores than the control group at both time $2(\mathrm{p}=0.008)$ and time $3(\mathrm{p}<0.001)$.

Conclusions and Implications: The SL course increased students' SE in teaching nutrition in the community, and other nutrition and dietetics programs may benefit from a similar SL course design. Researchers are currently validating the SET-NC survey in a nationwide population to provide a useful tool for measuring students' SE in teaching nutrition in the community.

Funding: None.

\section{Assessment of Cultural Knowledge and Knowledge and Skills Application in Dietetics Education}

Julie Plasencia, MS, RD, plasenc4@msu.edu, Michigan State University, 469 Wilson Road, Room 120, East Lansing, MI 48824; L. Weatherspoon, PhD, RD

Objective: The objective of this study was to assess among dietetic students if and how cultural competency knowledge and skills from a junior level cultural foods course are retained and applied in a senior nutrition course case study assignments using a cultural competency rubric.

Target Audience: IRB approval was obtained to recruit senior-level students from a capstone level course, Medical Nutrition Therapy (MNT)-II. Fifty-two students (57\%) consented; $37(71 \%)$ completed the cultural foods course in a prior year.

Theory, Prior Research, Rationale: Cultural competency is an important learning outcome for nutrition and 